\title{
Censoring Biological Echoes in Weather Radar Images
}

\author{
Valliappa Lakshmanan \\ University of Oklahoma \\ \& National Severe Storms Laboratory \\ Norman, Oklahoma, USA
}

\author{
Jian Zhang \\ National Severe Storms Laboratory \\ NOAA/Office of Atmospheric Research \\ Norman, Oklahoma, USA
}

\begin{abstract}
Weather radar data is susceptible to several artifacts due to anamalous propagation, ground clutter, electronic interference, sun angle, second-trip echoes and biological contaminants such as insects, bats and birds. Several methods of censoring radar reflectivity data have been devised and described in the literature. However, they all rely on analyzing the local texture and vertical profile of reflectivity fields.

The local texture of reflectivity fields suffices to remove most artifacts, except for biological echoes. Biological echoes have proved difficult to remove because they can have the same returned power and vertical profile as stratiform rain or snow.

In this paper, we describe a soft-computing technique based on clustering, segmentation and a two-stage neural network to censor all non-precipitating artifacts in weather radar reflectivity data. We demonstrate that the technique is capable of discrimination between light snow, stratiform rain and deep biological "bloom".
\end{abstract}

\section{Introduction}

Weather radar data are used operationally to warn of impending severe weather [1] and to create high-resolution precipitation estimates [2]. For example, [3] demonstrated that expected fatalities after Doppler radar installation in the United States were $45 \%$ lower and expected injuries were $40 \%$ lower. Radar data are routinely assimilated into numerical weather models and used for the prediction of convective systems [4].

All of these uses of weather radar require that echoes on radar correspond, broadly, to precipitation. By removing ground clutter contamination, rainfall from the radar data using the National Weather Service (NWS) Weather Surveillance Radar-Doppler 1998 (WSR-88D) can be improved [2], [5]. A large number of false positives for the Mesocyclone Detection Algorithm [6] are caused in regions of clear-air return [7], [8]. A hierarchical motion estimation technique segments and forecasts poorly in regions of ground clutter [9]. Hence, a completely automated algorithm that can remove regions of non-precipitating echo, such as ground clutter, anomalous propagation, radar artifacts and clear-air returns from the radar reflectivity field would be very useful in improving the performance of other automated weather radar algorithms.

[10] describe the causes, effects and characteristics of such contamination in weather radar data. [11], [12] determined individual features, and combinations of features, that can be used to remove range gates of radar reflectivity data that correspond to "bad echoes". Local neighborhoods in the vicinity of every range-gate in the three WSR-88D radar moments (reflectivity, velocity and spectrum width) were examined and used for automated removal of nonprecipitating echoes. [10] used a decision tree to classify range-gates into two categories - precipitation and nonprecipitation while [11] used a fuzzy rule base using features that included the SPIN feature introduced by [10]. [12] used a neural network to classify radar range gates into precipitation or non-precipitation, and followed the pixelwise classification with clustering. A cluster was censored if the majority of its pixels were determined to be nonprecipitating echo.

\subsection{Biological Echoes}

The methods of [10] and [11] worked well for anamalous propagation. When followed with the clustering-based postprocessing of [12], the quality of the resulting fields met the high threshold neccessary for fully automated quality control of radar data. However, biological contaminants can not be easily removed by means of such local texture or vertical profile features.

Biological echoes are difficult to discriminate from true precipitation because they share several characteristics of precipitating echoes. Biological contaminants (such as birds or bats) are moving, and therefore have non-zero Doppler velocities - the magnitude and texture of these scatters is very similar to that of widespread rain. Biological echoes have similar radar reflectivity values in the vertical and horizontal dimensions as snow or rain. As illustrated in Figure 1, from local characteristics alone, it is difficult to distinguish between biological artifacts and rain.

To discriminate between biological echoes and light rain/snow, it is necessary to consider the characteristics 

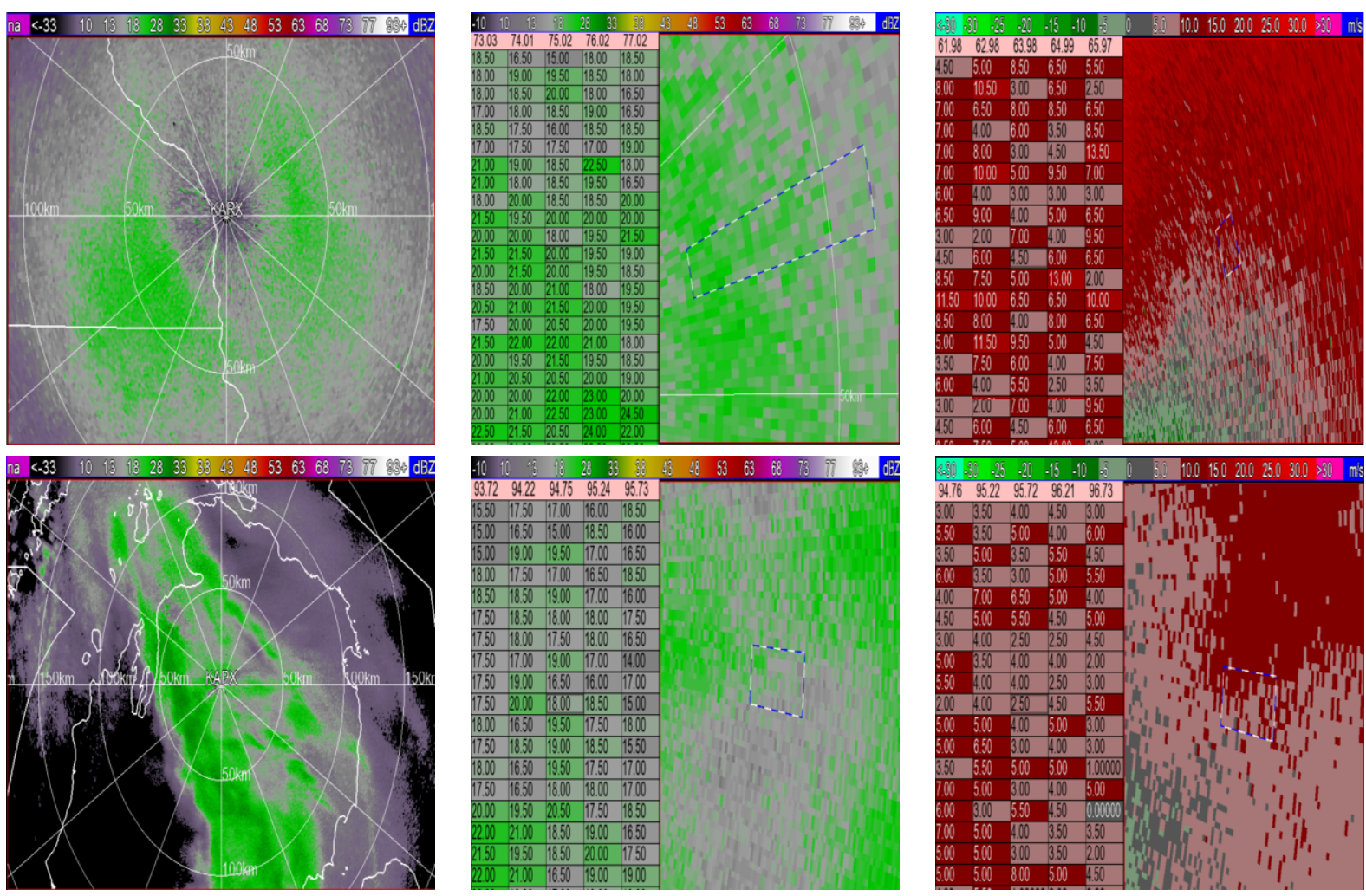

Figure 1. Why discriminating between biological echoes and light snow is hard. Top row: biological echoes; Bottom row: winter precipitation. Reflectivity (first two columns) and velocity (third column) from Doppler radar are shown. The snow case is from the KAPX radar on Jan. 17, 2009 at 19:23 UTC while the biological case is from the KARX radar on May 25, 2008 at 04:04 UTC.

of the entire echo, not just the vertical and horizontal neighborhood of a single pixel. Biological echoes tend to be circularly symmetric, centered around the radar and tend to drop in power as the distance from the radar increases (since the biological target fills less and less of the radar's sampling volume). These are tendencies, and not universally valid - storm cells may be circular, pass right over the radar and exhibit a very similar reflectivity profile. In addition, storm echoes may be embedded in an area of biological contamination, as shown in Figure 2d.

\section{Method}

Because biological echoes have a global profile that can be used to distinguish them from precipitating echoes, but all other artifacts need to be discriminated based on local characteristics, we followed the strategy of adding a feature to the local texture-based neural network that would be a probability that the pixel in question belongs to a biological echo.

To evaluate this probability, we computed several features and trained a neural network with one input, one hidden and a single output node. This output node's transfer function was a sigmoid, so that its output would correspond to the probability [13] that the cluster in question corresponded to biological echo.

To compute characteristics of biological echoes or "bloom", bloom features need to be computed. Naively clustering echoes based on contiguity will result in precipitation embedded within the bloom (such as in Figure 1c) also being considered part of the bloom and being potentially censored. To identify echo over radar as being bloom, the following steps are carried out:

1) Only range gates with an elevation within $4 \mathrm{~km}$ of the radar are considered, following studies carried out by [14] that indicated that this was where biological echoes are concentrated.

2) The reflectivity factor $(Z)$ values at constant azimuth are averaged

3) The values of $Z$ as it varies in range are fitted to line segments

4) The longest line segment (Pearson coefficient of 0.9 or better) whose slope is negative is considered to be the candidate bloom radius

Following the procedure outlined above takes into account the expected drop-off in returned intensity by range of 


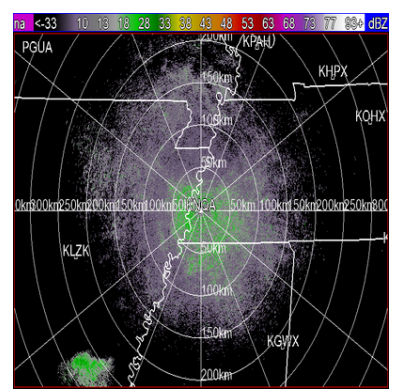

a

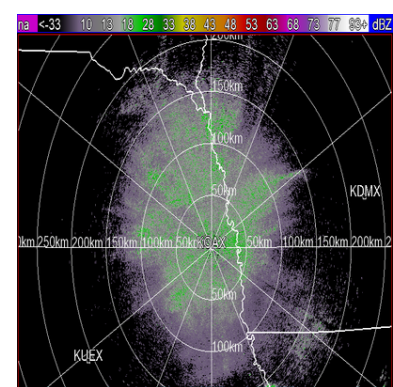

b

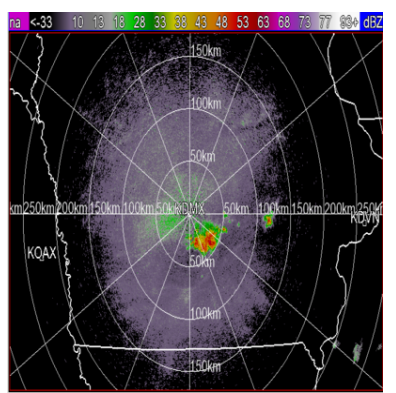

$\mathrm{c}$

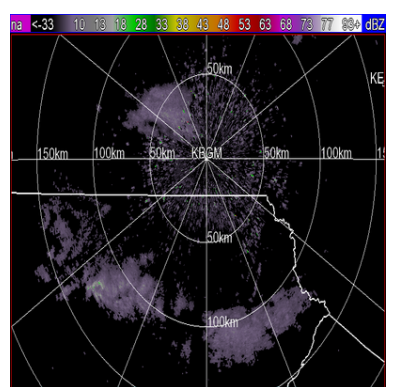

$\mathrm{d}$

Figure 2. (a,b): Examples of biological echoes: note that the characteristics of an entire echo may help identify regions in which biological echoes are more likely. (c) One difficulty: storms embedded in biological echo (d) Another difficulty: light snow moving over the radar. The data are from (a) KNQA on 2008/08/04 at 04:31 UTC (b) KOAX on 2008/08/05 at 03:18 UTC (c) KDMX on 2008/08/05 at 06:07 UTC and (d) KBGM on 2008/12/05 at 13:00 UTC

biological echoes while stopping the bloom detection when high reflectivities are encountered.

Once the bloom radius has been identified, several statistics are computed on the radar echoes within the bloom radius: (a) mean reflectivity (b) variance of reflectivity (c) symmetry of the mean of octants of the bloom (d) variance between the mean of the octants (e) fraction of the bloom that is filled with echo and (f) bloom radius. These features are used as inputs to a neural network that was trained to output the probability that the echo in question corresponds to bloom.

Each pixel in the radar scan is assigned a probability of bloom following a simple set of rules. Echoes within half-the-bloom radius are assigned the output probability of the neural network. Between half-the-bloom radius and the radius, low reflectivity echoes (defined as echoes of below a temperature-determined threshold that is $0 \mathrm{dBZ}$ in winter and $20 \mathrm{dBZ}$ in summer) are assigned the output probability of the neural network. Beyond the bloom radius, pixels are assigned the bloom probability only if they have low values and are connected to a pixel that has already been determined to be bloom.

The bloom probability result from the first neural network is thus assigned to every pixel in the image. This feature is provided as one of the inputs to a second, local-feature-based neural network. Thus second network had, as input features,: (1) Doppler velocity (2) mean of Doppler velocity (3) Standard deviation of Doppler velocity (4) Minimum standard deviation of Doppler velocity in neighborhood (5) Spectrum width (6) Reflectivity at lowest tilt (7) Neighborhood mean of reflectivity (8) Standard deviation of reflectivity (9) Minimum standard deviation of reflectivity in neighborhood (10) SPIN [10] (11) Inflections [11] (12) Reflectivity at second tilt (13) Mean reflectivity at second tilt (14) Difference between reflectivity value and mean (15) Minimum standard deviation (16) Maximum value in the vertical (17) VIL [15] (18) Difference between the two lowest tilts (19) Echo Top of $0 \mathrm{dBZ}$ (20) Echo Top of $20 \mathrm{dBZ}$ (21) Height of maximum (22) Fraction of neighborhood filled and (22) Probability that this pixel is part of a biological echo. The second neural network had 21 of the inputs chosen via feature selection as described in [12] and a 22nd determined by following image morphological operations on the result of the firststage neural network. The second-stage neural network was trained as in [12], and followed by the same cluster-based postprocessing followed in that paper. The output of the cluster-based postprocessing formed the final mask used to censor the reflectivity field.

\section{Results}

The training of the first-stage neural network (to perform the cluster-based discrimination between biological echoes and storm echoes) was carried out on a dataset consisting of 34 examples of good data around the data and 54 examples of biological artifacts. The good data points (which are scarce because we needed to find examples of storms directly over the radar) were repeated based on random selection so that the two classes had equal apriori probability. This dataset was divided 60:40 into a training and a validation dataset, and the validation dataset was used to carry out early stopping. The architecture of the neural network -1 hidden layer consisting of a single node - was set arbitrarily and the validation dataset was used to carry out early stopping. The ROC curve of the trained network on the validation set is shown in Figure 3a.

The probabilities from the first neural network formed the 22nd input to the second-stage neural network which operated on a pixel-by-pixel basis. This neural network was trained with much more data - nearly 1.5 million training patterns without velocity and more than 5 million patterns with velocity data. The ROC curve of the trained secondstage network on the validation set is shown in Figure $3 \mathrm{~b}$.

The technique described in this paper has been implemented and is being run in real-time to censor biological 


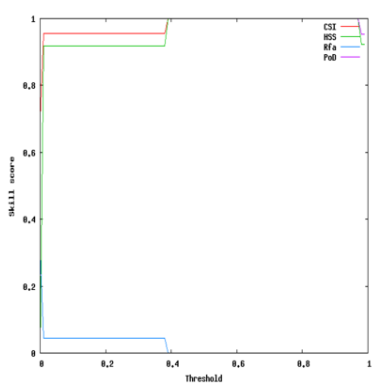

a

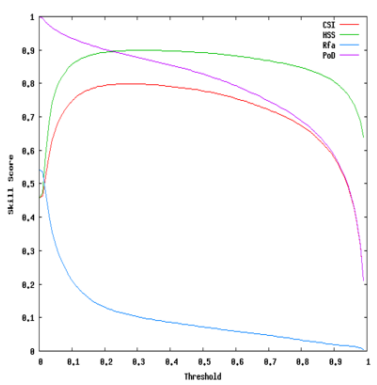

b

Figure 3. Measures of skill of (a) first-stage and (b) second-stage neural networks on their respective validation sets.

echoes in radar data. Some examples of the technique's performance on independent cases is shown in Figure 4. The following measures of skill are shown in the graph: Critical Success Index [16], Heidke Skill Score [17], Probability of Detection and Rate of False Alarm [18].

\section{Acknowledgment}

Funding for this research was provided under NOAA-OU Cooperative Agreement NA17RJ1227.

\section{References}

[1] D. H. Kitzmiller, W. E. McGovern, and R. F. Saffle, "The WSR-88D severe weather potential algorithm," Wea. Forecasting., vol. 10, pp. 141-159, 1995.

[2] R. Fulton, D. Breidenback, D. Miller, and T. O'Bannon, "The WSR-88D rainfall algorithm," Weather and Forecasting, vol. 13, pp. 377-395, 1998.

[3] K. Simmons and D. Sutter, "WSR-88D radar, tornado warnings, and tornado casualties," Weather and Forecasting, vol. 20 , no. 3 , pp. 301-310, 2005.

[4] J. Sun and J. Wilson, "The assimilation of radar data for weather prediction," Meteorological Monographs, vol. 30, no. 52, pp. 175-175, 2003.

[5] W. Krajewski and B. Vignal, "Evaluation of anomalous propagation echo detection in WSR-88D data: A large sample case study," J. Atmospheric and Oceanic Tech., vol. 18, pp. 807814, May 2001.

[6] G. Stumpf, A. Witt, E. D. Mitchell, P. Spencer, J. Johnson, M. Eilts, K. Thomas, and D. Burgess, "The national severe storms laboratory mesocyclone detection algorithm for the WSR-88D," Weather and Forecasting, vol. 13, no. 2, pp. 304326, 1998.

[7] K. McGrath, T. Jones, and J. Snow, "Increasing the usefulness of a mesocyclone climatology," in 21st Conference on Severe Local Storms, (San Antonio, TX), Amer. Meteor. Soc., 2002.
[8] R. J. Mazur, V. Lakshmanan, and G. J. Stumpf, "Quality control of radar data to improve mesocyclone detection," in Preprints, 20th Int'l Conf. on Inter. Inf. Proc. Sys. (IIPS) for Meteor., Ocean., and Hydr., (Seattle), p. P1.2, Amer. Meteor. Soc., Jan. 2004.

[9] V. Lakshmanan, R. Rabin, and V. DeBrunner, "Multiscale storm identification and forecast," J. Atm. Res., vol. 67, pp. 367-380, July 2003.

[10] M. Steiner and J. Smith, "Use of three-dimensional reflectivity structure for automated detection and removal of nonprecipitating echoes in radar data," J. Atmos. Ocea. Tech., vol. 19, no. 5, pp. 673-686, 2002.

[11] C. Kessinger, S. Ellis, and J. Van Andel, "The radar echo classifier: A fuzzy logic algorithm for the WSR-88D," in 3rd Conference on Artificial Applications to the Environmental Sciences, (Long Beach, CA), Amer. Meteor. Soc., Feb 2003.

[12] V. Lakshmanan, A. Fritz, T. Smith, K. Hondl, and G. J. Stumpf, "An automated technique to quality control radar reflectivity data," J. Applied Meteorology, vol. 46, pp. 288305, Mar 2007.

[13] C. Bishop, Neural Networks for Pattern Recognition. Oxford, 1995.

[14] S. Gauthreaux and C. Belser, "Displays of bird movements on the WSR-88D: Patterns and quantification," Wea. Forecasting, vol. 13, no. 2, pp. 453-464, 1988.

[15] D. R. Greene and R. A. Clark, "Vertically integrated liquid water - A new analysis tool," Mon. Wea. Rev., vol. 100, pp. 548-552, 1972.

[16] R. Donaldson, R. Dyer, and M. Kraus, "An objective evaluator of techniques for predicting severe weather events," in Preprints, Ninth Conf. on Severe Local Storms, (Norman, OK), pp. 321-326, Amer. Meteor. Soc., 1975.

[17] P. Heidke, "Berechnung des erfolges und der gute der windstarkvorhersagen im sturmwarnungsdienst," Geogr. Ann., vol. 8, pp. 301-349, 1926.

[18] D. Wilks, Statistical Methods in Atmospheric Sciences. Academic Press, 1995. 


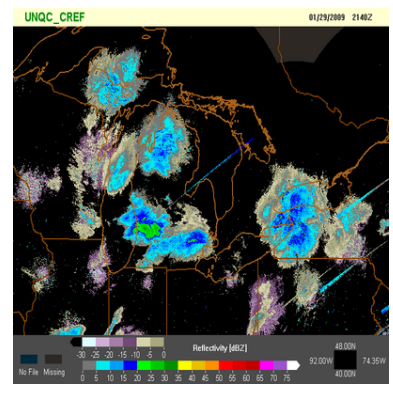

a

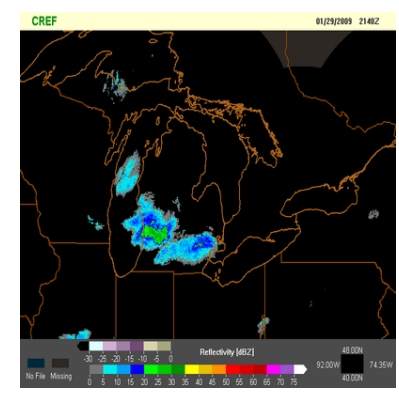

$\mathrm{b}$

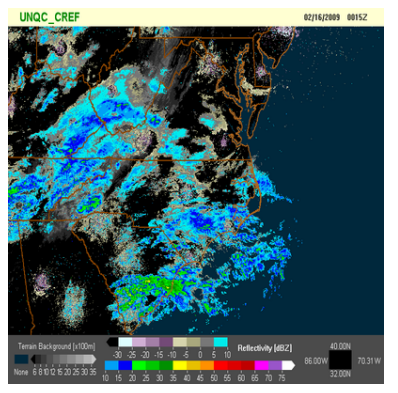

c

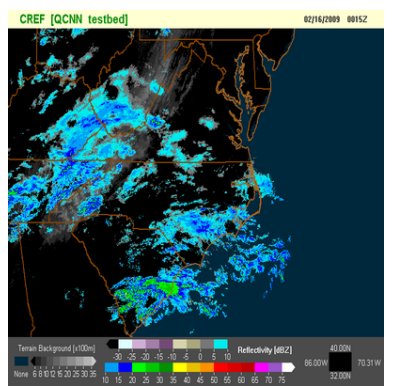

d

Figure 4. (a) Example of biological echo seen in real-time (b) Bloom removed; as can be seen, bloom from one of the radars has not been censored (c) Example of winter precipitation seen in real-time (d) All precipitation has been retained. 